\title{
Structural effects on carrier doping in carbon nanotube thin-film transistors
}

Yanlin $\mathrm{Gao}^{1, \text { a) }}$ and Susumu Okada ${ }^{1, \mathrm{~b})}$

Graduate School of Pure and Applied Sciences, University of Tsukuba, 1-1-1 Tennodai, Tsukuba, Ibaraki 305-8571, Japan

(Dated: 25 June 2020)

Based on density functional theory, we studied the electronic properties of carbon nanotube (CNT) thin films under an external electric field. The carrier accumulation resulting from an electric field depends slightly on the CNT species that form the thin films and their arrangement with respect to the electrode. Although most of the carriers are accumulated in the CNTs located at the electrode side, wave function hybridization between semiconducting CNTs slightly enhances the carrier penetration into the opposite CNT layer. Metallic CNTs strongly depress or enhance the carrier penetration for the thin films when they are located at the electrode side or not, respectively.

a) Electronic mail: ylgao@comas.frsc.tsukuba.ac.jp

b)Electronic mail: sokada@comas.frsc.tsukuba.ac.jp 


\section{INTRODUCTION}

Carbon nanotubes $(\mathrm{CNTs})^{1}$ have attracted much attention in both fundamental and applied sciences because of their geometric and electronic structures. Rigid covalent tubular networks of $\mathrm{sp}^{2} \mathrm{C}$ atoms cause a high Young's modulus ${ }^{2-5}$, which results in a remarkable thermal conductivity ${ }^{6-8}$. Owing to the nanoscale tubular space inside the covalent networks, CNTs can accommodate foreign atoms and molecules that possess different condensed structures from their bulk forms. This results in interesting hybrid systems whose physical/chemical properties are different from the simple sum of each constituent unit ${ }^{9-11}$. Moreover, CNTs occasionally form bundle structures, in which CNTs are packed in hexagonal form owing to the strong van der Waals interaction arising from their one-dimensional structures. The covalent tubular structure and structural hierarchy endow a CNT and its derivatives with unusual electronic structures. An individual CNT is either a metal or semiconductor depending on the boundary conditions imposed on its honeycomb networks

along its circumference ${ }^{12-14}$. A morphological hierarchy of a CNT and its hybrids causes further variation in its electronic structures: Intertube interaction causes a tiny pseudogap at the Fermi level on bundles consisting of metallic $\mathrm{CNTs}^{15-17}$ and on double-walled metallic $\mathrm{CNTs}^{18,19}$.

These unusual physical properties allow CNTs to be useful materials in various modern technologies, such as electronic, mechanical, thermal, and energy devices. Among these applications, it has been demonstrated that CNTs work as conducting channels in field effect transistors $(\mathrm{FETs})^{20-28}$ and interconnects in semiconductor electronic devices ${ }^{29,30}$, owing to their semiconducting and metallic electronic structures, respectively. In usual CNTbased FETs, the channels consist of mat films of CNTs, which intrinsically intersect or align with other CNTs. At the intersectional and aligned regions, the intertube interaction plays decisive roles to determine the device performance, because the intertube interaction basically causes modulation of the electronic structure of the CNTs. Although the CNT mat films and aligned CNTs can work as a conducting channel in real devices, such as multi-stage ring oscillators ${ }^{24,25}$ and thermoelectric devices ${ }^{31-33}$, little is known about the microscopic mechanism of carrier accumulation in CNT thin films by a gate electrode. In our previous work, we predicted that field inversion occurs at the intersectional region of two CNTs under a weak electric field ${ }^{34,35}$. However, it is still uncertain whether the accumulated 
carrier distribution in densely aligned CNT thin films in the FET structure depends on the external electric field and the CNT arrangement or not.

Accordingly, in this manuscript, we aim to elucidate the carrier distribution in CNT thin films, which are simulated by a bilayer of CNTs with different diameters and intertube spacings, under an external electric field that injects electrons/holes into the CNT thin films. Our calculations based on density functional theory (DFT) show that the distribution of accumulated carriers by the electric field slightly depends on the field strength, constituent CNT species, and intertube spacing. Injected carriers are highly concentrated in the CNT layer on the electrode side under a strong electric field. A large intertube spacing also causes the carrier concentration owing to the absence of intertube orbital hybridization. In contrast, a small intertube spacing and the weak external field enhance the carrier penetration into the second CNT layer. We also found that metallic CNT arrangements substantially affect the carrier distribution in CNT thin films.

\section{CALCULATION METHODS}

All calculations were performed within the framework of DFT ${ }^{36,37}$ implemented in a STATE package ${ }^{38}$. To express the exchange correlation potential among interacting electrons, we used the local density approximation (LDA) with a functional form fitted to Monte Carlo results for a homogeneous electron gas generated by Perdew and Zunger ${ }^{39,40}$, because the LDA is known to qualitatively reproduce the interlayer spacing of $\mathrm{sp}^{2} \mathrm{C}$ materials. We used an ultrasoft pseudopotential to describe the interactions between the valence electrons and ions generated by the Vanderbilt scheme ${ }^{41}$. The valence wave function and deficit charge density were expanded in terms of the plane-wave basis set with cutoff energies of 25 and 225 Ry, respectively, which provide sufficient convergence in the geometries and electronic structures of carbon related materials ${ }^{42,43}$. Integration over the Brillouin zone was carried out using $6 \times 2 \times 1 \boldsymbol{k}$-meshes for the directions along, across, and normal to the CNTs with a film structure. We adopted the effective screening medium method (ESM) ${ }^{44}$ to solve Poisson's equation including the excess electrons or holes injected by the electrode within the framework of DFT with the plane-wave basis set.

We considered CNT thin-film FETs consisting of bilayer CNTs situated in front of a planar gate electrode simulated by an effective screening medium with the infinite permittivity 


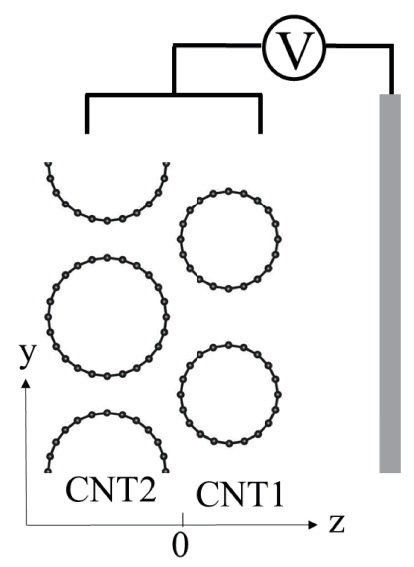

FIG. 1. Structural models of CNT thin-film (CNT1/CNT2) FET consisting of CNT1 and CNT2 layers situated in front of a planar gate electrode. Circles and gray rectangle denote carbon atoms and the planar gate electrode, respectively.

mimicking an ideal metal electrode (Fig. 1). In the thin films, CNTs were arranged in a parallel manner with a CNT spacing of 3 and $10 \AA$ to investigate the influence of CNT density on the carrier accumulation. We considered $(10,0),(11,0)$, and $(12,0)$ CNTs with the arrangement of $\mathrm{CNT} 1 / \mathrm{CNT} 2$, where CNT1 was located at the electrode side with a vacuum spacing of approximately $6 \AA$ while CNT2 was located below CNT1 with a spacing of $3 \AA$ or more. An electric field was applied between the electrode and the CNTs under the gate voltages of $\mathrm{V}_{G}= \pm 1.8 \mathrm{~V}$ and $\pm 0.18 \mathrm{~V}$, which corresponded to the injection of 0.1 and 0.01 electrons/holes into CNT thin films, respectively. The atomic structures were optimized until the force acting on each atom was less than $5 \mathrm{meV} / \AA$ under the fixed lattice parameters along the tube axis of $4.26 \AA$ and without the external electric field. During the calculations under the conditions with a finite electric field, the geometries were kept the same as those with a zero electric field.

\section{RESULTS AND DISCUSSION}

Figure 2 shows the accumulated carrier density in the CNT thin films in which the CNTs were separated by approximately $3 \AA$ corresponding with the van der Waals spacing. The injected carriers were primarily distributed in the CNT1 layer situated at the electrode side, irrespective of the CNT species, carrier concentration, and carrier species (Table I). For 
(a)

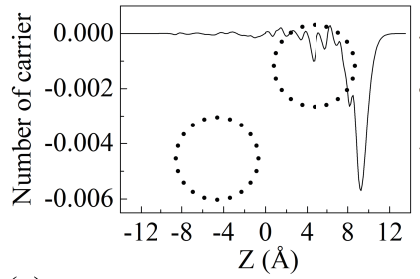

(c)

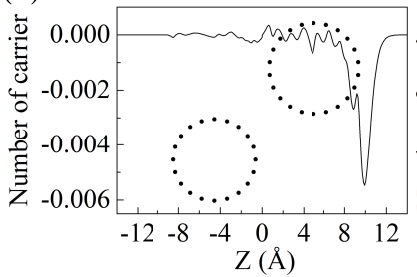

(e)

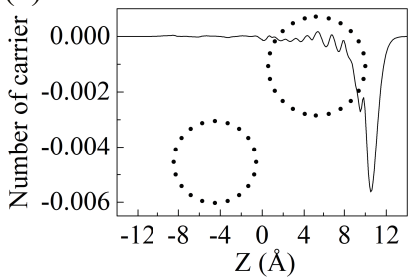

(g)

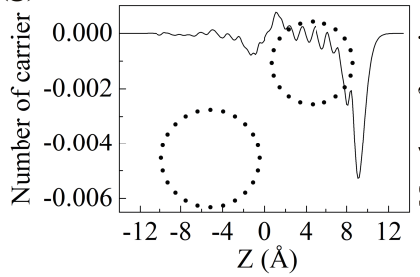

(i)

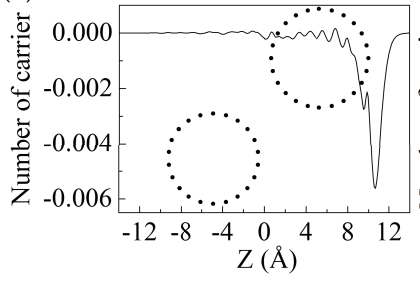

(b)
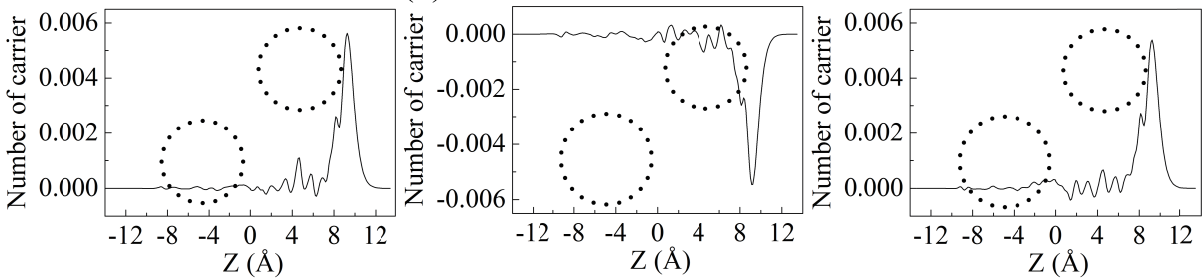

(d)
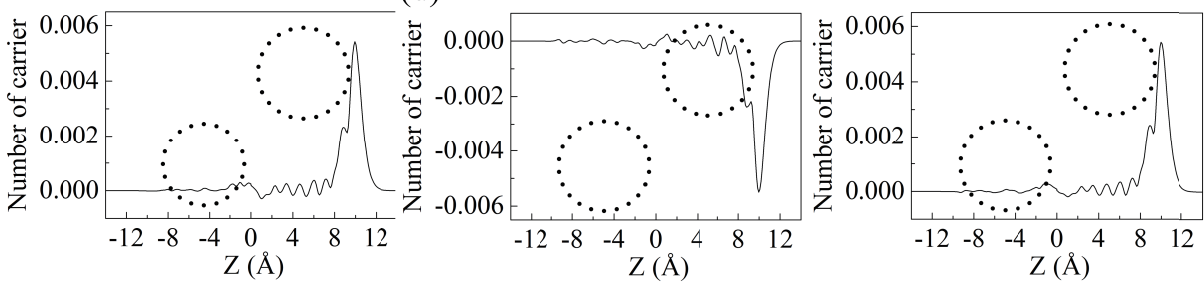

(f)
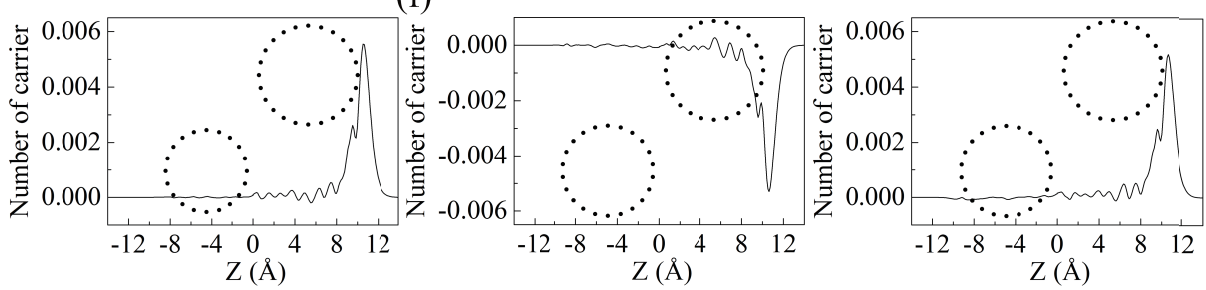

(h)
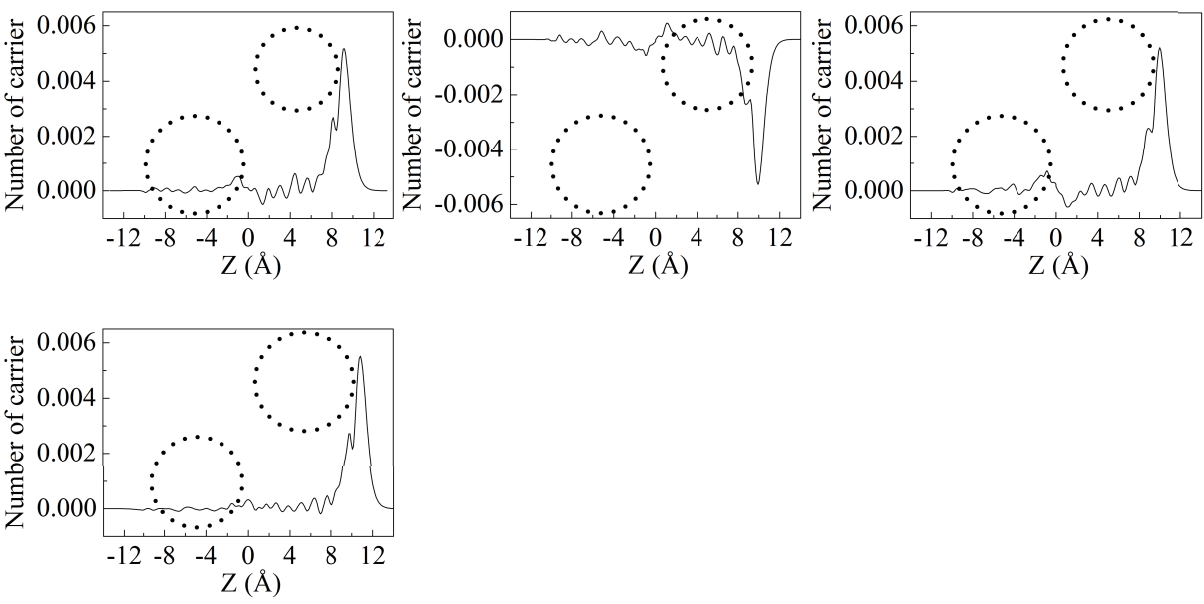

FIG. 2. Plane averaged carrier density as a function of $z$ corresponding to the normal direction to the electrode in densely packed (a) $(10,0) /(10,0)$, (b) $(10,0) /(11,0)$, (c) $(11,0) /(10,0)$, (d) $(11,0) /(11,0),(\mathrm{e})(12,0) /(10,0)$, (f) $(12,0) /(11,0),(\mathrm{g})(10,0) /(12,0),(\mathrm{h})(11,0) /(12,0)$, and (i) $(12,0) /(12,0)$ CNT thin films under an external electric field. The electrode is situated at the right end of each figure. In each figure, left and right panels correspond to the carrier densities under $0.1 \mathrm{~h}$ and $0.1 \mathrm{e}$ doping, respectively. 
TABLE I. Concentration of accumulated carrier on the CNT1 layer of the CNT thin films with various arrangements under high and low carrier doping.

\begin{tabular}{ccccc|ccc}
\hline CNT1/CNT2 & \multicolumn{3}{c}{$\mathrm{d}=3 \AA[\%]$} & \multicolumn{3}{c}{$\mathrm{d}=10 \AA[\%]$} \\
& $0.1 \mathrm{e}$ & $0.1 \mathrm{~h}$ & $0.01 \mathrm{e}$ & $0.01 \mathrm{~h}$ & $0.1 \mathrm{e}$ & $0.1 \mathrm{~h}$ \\
\hline$(10,0) /(10,0)$ & 98 & 98 & 98 & 99 & 99 & 100 \\
$(10,0) /(11,0)$ & 94 & 96 & 100 & 93 & 100 & 100 \\
$(11,0) /(10,0)$ & 94 & 96 & 82 & 76 & 96 & 100 \\
$(11,0) /(11,0)$ & 95 & 96 & 89 & 91 & 100 & 100 \\
$(12,0) /(10,0)$ & 99 & 100 & 100 & 100 & 100 & 100 \\
$(12,0) /(11,0)$ & 100 & 99 & 100 & 77 & 100 & 100 \\
$(10,0) /(12,0)$ & 92 & 84 & 80 & 80 & 80 & 75 \\
$(11,0) /(12,0)$ & 88 & 90 & 83 & 88 & 74 & 75 \\
$(12,0) /(12,0)$ & 99 & 100 & 100 & 100 & 100 & 100 \\
\hline
\end{tabular}

the thin films consisting of semiconducting CNTs under high carrier doping, approximately $95 \%$ of carriers were accommodated in the CNT1 layer for both electron and hole doping. Furthermore, the distribution was highly concentrated at the electrode side wall of CNT1. This distribution was basically independent of the CNT arrangement with respect to the electrode [Figs. 2(a)- 2(d)]. In contrast, for the thin films containing a metallic CNT, the distribution of accumulated carriers strongly depended on the CNT arrangements whether the metallic $(12,0)$ CNT was located at the electrode side or not: The carrier was perfectly localized on CNT1, when the metallic CNT layer was located at the electrode side [Figs. 2(e) and $2(\mathrm{f})]$. On the other hand, the carrier significantly penetrated into the CNT2 layer for the thin films where the metallic CNT was in the second layer [Figs. 2(g) and 2(h)]. For thin films consisting only of metallic CNTs, the carrier was perfectly localized on the CNT1 [Fig. 2(i)].

The decrease of the doping amount affected the distribution in some cases [Fig. 3 ]. For the thin films consisting of heterogeneous CNTs, the accumulated carrier exhibited asymmetric properties with respect to the CNT arrangements: The carrier substantially penetrated to the second layer under the $(11,0) /(10,0)$ arrangement, and 19 and $24 \%$ of the electron and hole, respectively, penetrated into the CNT2 layer [Fig. 3(c)]. In contrast, 
in the thin film of $(10,0) /(11,0)$, the carrier penetration was small and absent in the cases of hole and electron doping, respectively [Fig. 3(b)]. This anomalous carrier penetration is ascribed to the band edge alignment of the thin film with respect to CNT arrangement. For the $(11,0) /(10,0)$ thin film, the band edges are primarily contributed from the state associated with $(11,0) \mathrm{CNT}$. The carrier penetration was also enhanced for the thin films containing metallic $(12,0) \mathrm{CNT}$, which were arranged in the second layer. This anomalous carrier penetration is ascribed to the band edge aligment of the thin film with respect to the CNT arrangement. For the $(11,0) /(10,0)$ thin film, the band edges are primarily contributed from the state associated with (11,0) CNT. Indeed, $20 \%$ of the electron and hole penetrated to the second layer consisting of $(12,0)$ CNTs for the $(10,0) /(12,0)$ thin film [Fig. 3(g)], and $12 \%$ of the hole and $18 \%$ of the electron penetrated in the second metallic layer for the $(11,0) /(12,0)$ thin film [Fig. 3(h)]. These facts implied that the carrier distribution in the monolayer CNT thin film is qualitatively different from that in the thin film comprising of two layers. In contrast, because the carrier penetration in second layer is considerably small, similar distribution of accumulated carrier is expected in thicker thin film.

The carrier distribution was further modulated by the CNT density in the thin films. Figure 4 shows the accumulated carrier density in CNT thin films in which the CNT was separated by approximately $10 \AA$ under the high carrier doping (0.1e and $0.1 \mathrm{~h})$ (Table I). For most of the thin films, the injected electron and hole were further concentrated in the CNT1 layer compared with the thin films in which CNTs were packed with the van der Waals spacing. For the thin films consisting of semiconducting CNTs, electrons and holes were perfectly accommodated in the CNT1 layer, except the $(11,0) /(10,0)$ thin films under electron doping. In addition, the thin film consisting only of the metallic CNT also exhibited perfect carrier localization on CNT1 layer. For the thin films containing semiconducting and metallic CNTs, both electron and hole were perfectly localized on the CNT1 layer, when the metallic CNT was located at the electrode side. When the metallic CNT was located at the opposite to the electrode, carrier penetration into the metallic CNT layer was substantially enhanced compared with the case of the thin films in which CNTs were packed with the van der Waals spacing. In this case, 20 25\% of the carriers spilled out of the CNT2 layer.

The influence of the CNT spacing on carrier distribution under an electric field implied that the intralayer and interlayer wave function hybridization plays decisive roles to determine the distribution in terms of the CNT species and arrangements. Figure 5 shows the 
electronic energy band and the wave functions associated with the band edges of the CNT thin films where CNTs were separated from each other by the van der Waals spacing. In the thin films consisting of semiconducting CNTs, the wave functions of the valence and conduction band edges exhibited a hybridized nature not only in the layer but also between layers. Thus, the hybrid nature of the valence and conduction band edges caused small but significant charge penetration into the CNT2 layer. However, the wave function at the Fermi level of the thin films containing $(12,0) \mathrm{CNT}$ was approximately localized on the metallic CNT. Therefore, the accumulated carrier distribution strongly depends on the $(12,0) \mathrm{CNT}$ arrangement with respect to the electrode: The carrier was perfectly localized on $(12,0) \mathrm{CNT}$ when they formed on the electrode side layer, while substantial penetration occurred when they formed on the second layer.

Increasing the intertube spacing within the layers led to the increase of the intertube spacing between layers. Accordingly, the wave function overlap between adjacent CNTs was substantially suppressed, which enabled recovery of their isolated electronic structure. Indeed, the wave function associated with the band edges or the Fermi level was perfectly localized on the corresponding CNT without sowing any hybridized nature. It is worth noting that the wave function of all states of the thin films consisting of homogeneous CNTs was extended throughout the thin films. The carrier concentration in the topmost layer was ascribed to the isolated nature of the wave function as well as the band edge shift caused by the electrostatic potential shift.

Figure 6 shows the electrostatic potential across the CNT thin films under an electric field. The potential was evaluated by taking the difference between that under the field and that without the field, to exclude the steep potential valleys associated with nuclei. Because of the small carrier penetration into the CNT2 layers of the thin films consisting of semiconducting CNTs, the electric field only appeared between the electrode and the CNT1 layer for both electron and hole doping. In contrast, for the thin films of $(11,0) /(12,0)$ and $(10,0) /(12,0)$, because of the substantial charge accumulation in the $(12,0)$ CNT layer, a substantial potential gradient between the CNT2 and CNT1 layers, in addition to a strong field outside the films, were observed. For the thin films solely consisting of metallic CNTs, the high carrier concentration in the CNT1 layer caused the field inversion between the CNT1 and CNT2 layers with respect to the applied external field. 


\section{CONCLUSION}

We studied the electronic structures of CNT thin films, simulated by parallel-aligned CNTs, under an external electric field using DFT combined with ESM. Our calculations show that the CNT species and their arrangement in the thin films affect the carrier accumulation by an electric field. For the thin films consisting only of semiconducting CNTs under a high carrier concentration or large gate voltage, the carriers are primarily distributed on the CNT layer situated at the electrode side with a small carrier penetration into the opposite CNT layer. In contrast, the carrier penetration in the second CNT layer is enhanced when the thin films are under a low carrier doping or a low gate voltage. For the thin films containing metallic CNTs, accumulated carrier distribution strongly depends on the metallic CNT arrangement with respect to the electrode: The injected carrier is perfectly localized on the metallic CNTs when they are situated at the electrode side, while the carrier substantially penetrates into CNT2 layer under the opposite arrangement. In addition to the carrier concentration, CNT spacing in the thin films further affect the carrier distribution. A large intertube spacing in the thin films, where each CNT is regarded as an isolated CNT, substantially enhances the carrier concentration on CNT1, except in the thin films containing metallic CNTs. The thin films containing metallic CNTs exhibit a similar carrier distribution in the thin films where the CNTs are densely packed with each other. Wave functions associated with the band edges of the thin films indicate that the carrier penetration in the thin films, consisting only of semiconducting CNTs, is ascribed to the large wave function hybridization between adjacent CNTs.

\section{ACKNOWLEDGMENTS}

This work was supported by JST-CREST Grant Numbers JPMJCR1532 and JPMJCR1715 from the Japan Science and Technology Agency, JSPS KAKENHI Grant Numbers JP17H01069, JP16H00898, and JP16H06331 from the Japan Society for the Promotion of Science, and the Joint Research Program on Zero-Emission Energy Research, Institute of Advanced Energy, Kyoto University. Part of the calculations was performed on an NEC SX-Ace at the Cybermedia Center at Osaka University and on an SGI ICE XA/UV at the Institute of Solid State Physics, The University of Tokyo. 
The data that support the findings of this study are available from the corresponding author upon reasonable request.

\section{REFERENCES}

${ }^{1}$ S. Iijima, Nature 354, 56 (1991).

${ }^{2}$ R. S. Ruoff and D. C. Lorents, Carbon 33, 925 (1995).

${ }^{3}$ M. M. J. Treacy, T. W. Ebbesen, and J. M. Gibson, Nature 381, 678 (1996).

${ }^{4}$ M.-F. Yu, O. Lourie, M. J. Dyer, K. Moloni, T. F. Kelly, and R. S. Ruoff, Science 287, 637 (2000).

${ }^{5}$ M.-F. Yu, B. S. Files, S. Arepalli, and R. S. Ruoff, Phys. Rev. Lett. 84, 5552 (2000).

${ }^{6}$ S. Berber, Y.-K. Kwon, and D. Tománek, Phys. Rev. Lett. 84, 4613 (2000).

${ }^{7}$ T. Yamamoto, S. Watanabe, and K. Watanabe, Phys. Rev. Lett. 92, 075502 (2004).

${ }^{8}$ T. Yamamoto and K. Watanabe, Phys. Rev. Lett. 96, 255503 (2006).

${ }^{9}$ B. W. Smith, M. Monthioux, and D. E. Luzzi, Nature 396, 323 (1998).

${ }^{10}$ S. Okada, S. Saito, and A. Oshiyama, Phys. Rev. Lett. 86, 3835 (2001).

${ }^{11}$ M. Monthioux, Carbon 40, 1809 (2002).

${ }^{12}$ N. Hamada, S.-I. Sawada, and A. Oshiyama, Phys. Rev. Lett. 68, 1579 (1992).

${ }^{13}$ R. Saito, M. Fujita, M. S. Dresselhaus, and G. Dresselhaus, Appl. Phys. Lett. 60, 2204 (1992).

${ }^{14}$ K. Tanaka, K. Okahara, M. Okada, and T. Yamabe, Chem. Phys. Lett. 191, 469 (1992).

${ }^{15}$ P. Delaney, H. J. Choi, J. Ihm, S. G. Louie, and M. L. Cohen, Nature, 391, 466 (1998).

${ }^{16}$ Y.-K. Kwon, S. Saito, and D. Tománek, Phys. Rev. B 65, 041402R (1998).

${ }^{17}$ P. Delaney, H. J. Choi, J. Ihm, S. G. Louie, and M. L. Cohen, Phys. Rev. B 60, 7899 (1999).

${ }^{18}$ Y.-K. Kwon and D. Tománek, Phys. Rev. B 58, R16001 (1998).

${ }^{19}$ Y. Miyamoto, S. Saito, and D. Tománek, 65, 041402R (2001).

${ }^{20}$ S. J. Tans, A. R. M. Verschueren, and C. Dekker, Nature 393, 49 (1998).

${ }^{21}$ R. Martel, T. Schmidt, H. R. Shea, T. Hartel, and Ph. Avouris, Appl. Phys. Lett. 73, 2447 (1998).

${ }^{22}$ Y. Nosho, Y. Ohno, S. Kishimoto, and T. Mizutani, Appl. Phys. Lett. 86, 073105 (2005).

${ }^{23}$ Y. Nosho, Y. Ohno, S. Kishimoto, and T. Mizutani, Nanotechnology 17, 3412 (2006). 
${ }^{24}$ D.-M. Sun, M. Y. Timmermans, Y. Tian, A. G. Nasibulin, E. I. Kauppinen, S. Kishimoto, T. Mizutani, and Y. Ohno, Nat. Nanotechnol. 6, 156 (2011).

${ }^{25}$ D.-M. Sun, M. Y. Timmermans, A. Kaskela, A. G. Nasibulin, S. Kishimoto, T. Mizutani, E. I. Kauppinen, and Y. Ohno, Nat. Commun. 4, 2302 (2013).

${ }^{26}$ M. Chen, W. Li, A. Kumar, G. Li, M. E. Itkis, B. M. Wong, and E. Bekyarova, ACS Appl. Mater. interfaces. 11, 19315-19323 (2019).

${ }^{27}$ P. M. Rajanna, S. Luchkin, K. V. Larionov, A. Grebenko, Z. I. Popov, P. B. Sorokin, M. Danilson, S. Bereznev, P. D. Lund, and A. G. Nasibulin, J. Phys. Chem. Lett. 11, 504-509 (2020).

${ }^{28}$ A. C. Ford, M. Shaughnessy, B. M. Wong, A. A. Kane, O. V. Kuznetsov, K. L. Krafcik, W. E. Billups, R. H. Hauge, and F. Leonard, Nanotechnology 24, 105202 (2013).

${ }^{29}$ M. Nihei, D. Kondo, A. Kawabata, S. Sato, H. Shioya, M. Sakaue, T. Iwai, M. Ohfuti, and Y. Awano, IEEE International Interconnect Technology Conference, Tech. Dig. 234 (2005).

${ }^{30}$ S. Sato M. Nihei, A. Mimura, A. Kawabata, D. Kondo, H. Shioya, T. Iwai, M. Mishima, M. Ohniti, and Y. Awano, IEEE International Interconnect Technology Conference, Tech. Dig. 2302006.

${ }^{31}$ Y. Nakai, K. Honda, K. Yanagi, H. Kataura, T. Kato, T. Yamamoto, and Y. Maniwa, Appl. Phys. Express 7, 025103 (2014).

${ }^{32}$ K. Fukuhara, Y. Ichinose, H. Nishidome, Y. Yomogida, F. Katsutani, N. Komatsu, W. Gao, J Kono, and K. Yanagi, Appl. Phys. Lett. 113, 243105 (2018)

${ }^{33}$ Y. Ichinose, A. Yoshida, K. Horiuchi, K. Fukuhara, N. Komatsu, W. Gao, Y. Yomogida, M. Matsubara, T. Yamamoto, J. Kono, and K. Yanagi, Nano Lett. 19, 7370 (2019).

${ }^{34}$ T. Kochi and S. Okada, Appl. Phys. Express 9, 085103 (2016).

${ }^{35}$ T. Kochi and S. Okada, Appl. Phys. Express 10, 075101 (2017).

${ }^{36}$ P. Hohenberg and W. Kohn, Phys. Rev. 136, B864 (1964).

${ }^{37}$ W. Kohn and L. J. Sham, Phys. Rev. 140, A1133 (1965).

${ }^{38}$ Y. Morikawa, K. Iwata, and K. Terakura, Appl. Surf. Sci. 169-170, 11 (2000).

${ }^{39}$ J. P. Perdew and A. Zunger, Phys. Rev. B 23, 5048 (1981).

${ }^{40}$ D. M. Ceperley and B. J. Alder, Phys. Rev. Lett. 45, 566 (1980).

${ }^{41}$ D. Vanderbilt, Phys. Rev. B 41, 7892 (1990).

${ }^{42}$ M. Maruyama and S. Okada, Appl. Phys. Express 6, 095101 (2013). 
${ }^{43}$ M. Maruyama, N. T. Cuong, and S. Okada, J. Phys. Soc. Jpn. 84, 084706 (2015).

${ }^{44}$ M. Otani and O. Sugino, Phys. Rev. B 73, 115407 (2006). 
(a)

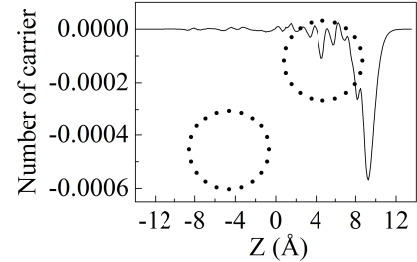

(c)

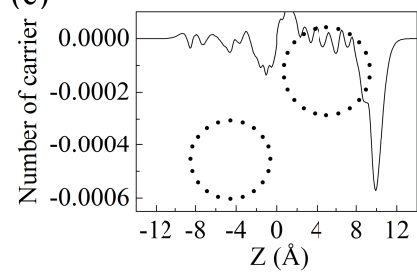

(e)

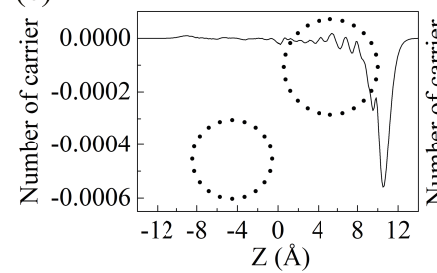

(g)

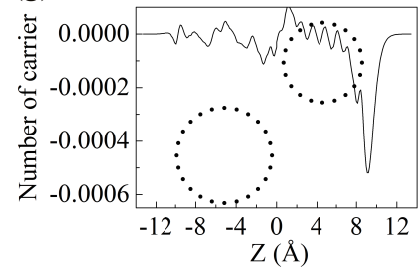

(i)
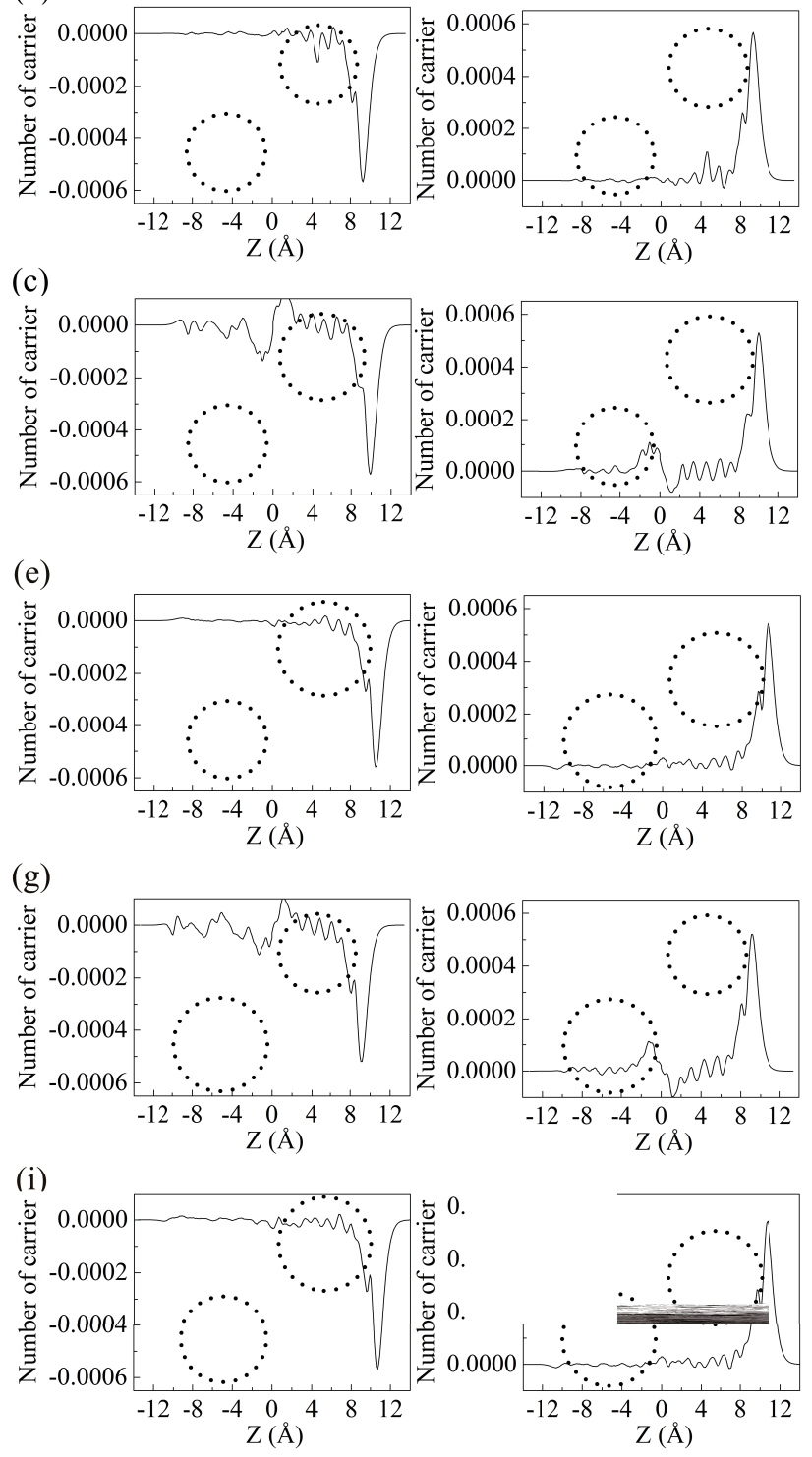

(b)
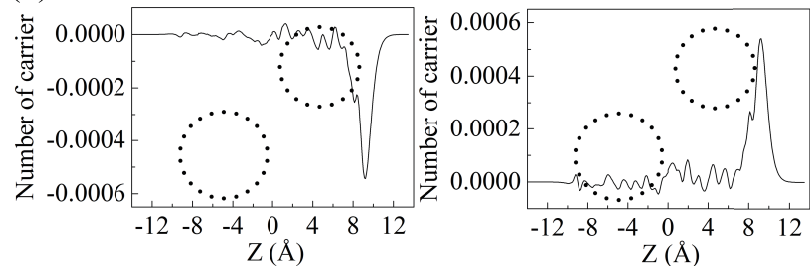

(d)

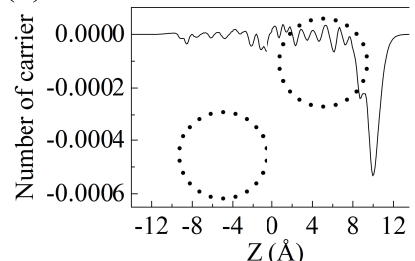

(t)

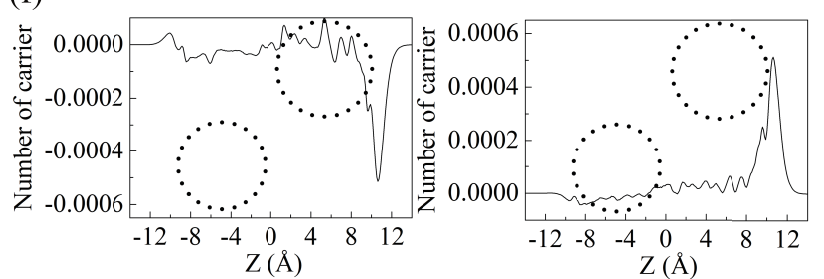

(h)
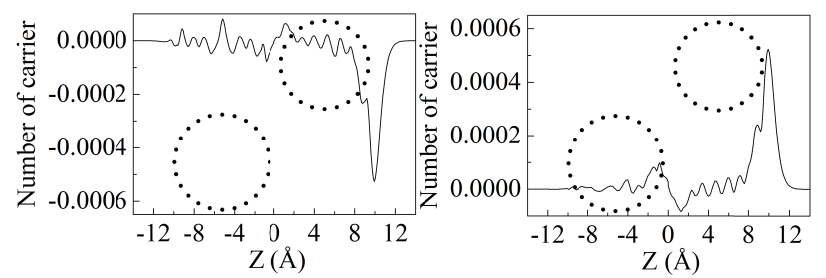

FIG. 3. Plane averaged carrier density as a function of $z$ corresponding to the normal direction to the electrode in densely packed (a) $(10,0) /(10,0)$, (b) $(10,0) /(11,0)$, (c) $(11,0) /(10,0)$, (d) $(11,0) /(11,0)$, (e) $(12,0) /(10,0)$, (f) $(12,0) /(11,0)$, (g) $(10,0) /(12,0),(\mathrm{h})(11,0) /(12,0)$, and (i) $(12,0) /(12,0)$ CNT thin films under an external electric field. The electrode is situated at the right end of each figure. In each figure, left and right panels correspond to the carrier densities under $0.01 \mathrm{~h}$ and $0.01 \mathrm{e}$ doping, respectively. 
(a)

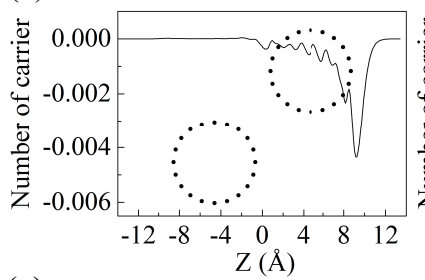

(c)

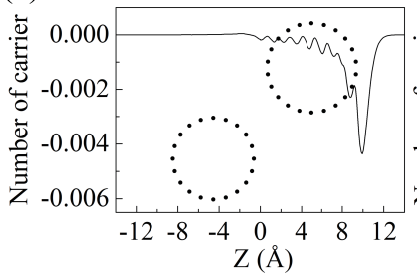

(e)

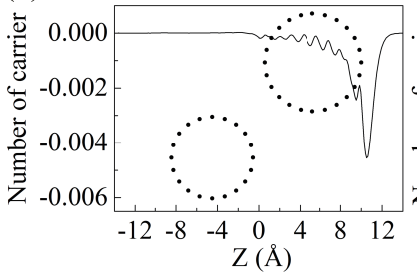

(g)

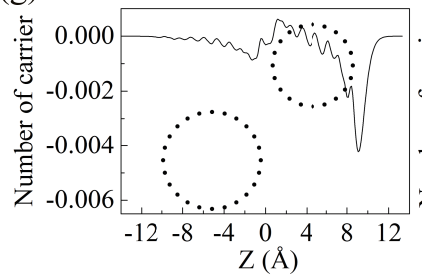

(i)
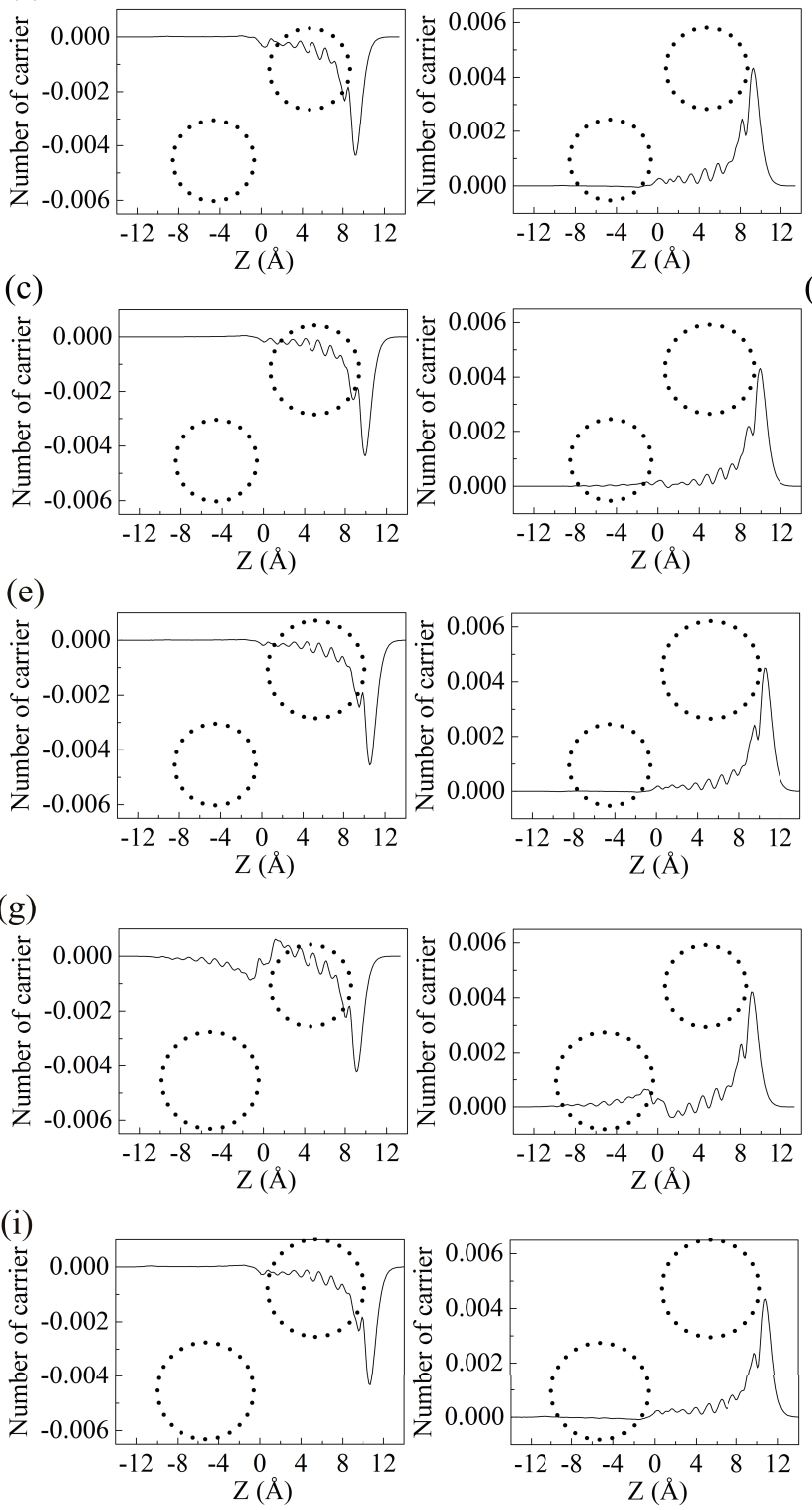

(b)
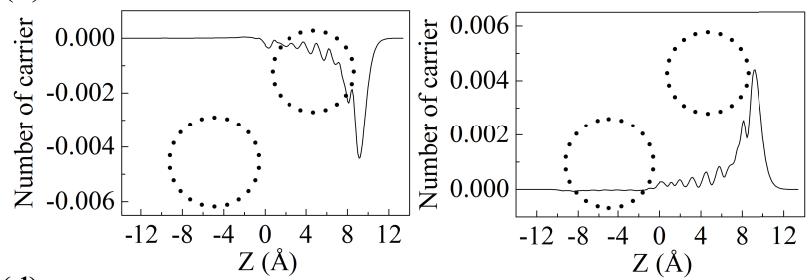

(d)
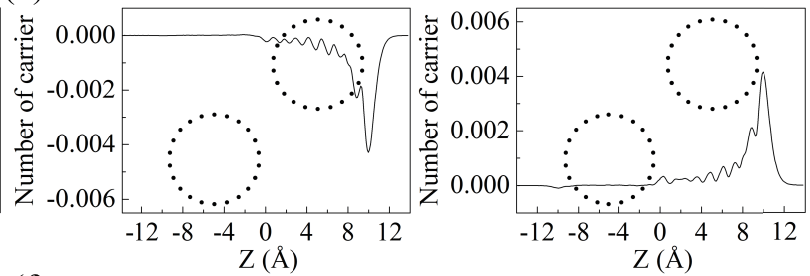

(f)

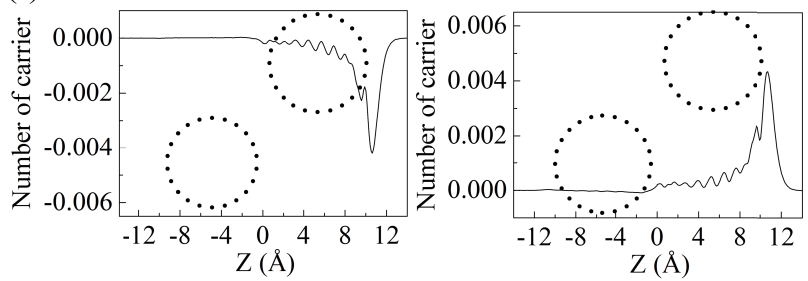

(h)

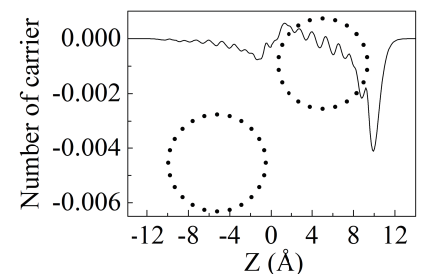

FIG. 4. Plane averaged carrier density as a function of $z$ corresponding to the normal direction to the electrode in $(\mathrm{a})(10,0) /(10,0)$, (b) $(10,0) /(11,0)$, (c) $(11,0) /(10,0)$, (d) $(11,0) /(11,0)$, (e) $(12,0) /(10,0)$, (f) $(12,0) /(11,0),(\mathrm{g})(10,0) /(12,0),(\mathrm{h})(11,0) /(12,0)$, and $(\mathrm{i})(12,0) /(12,0)$ CNT thin films, where CNT is separated by $1 \mathrm{~nm}$ from an adjacent CNT, under an external electric field. The electrode is situated at the right end of each figure. In each figure, left and right panels correspond to the carrier densities under $0.1 \mathrm{~h}$ and $0.1 \mathrm{e}$ doping, respectively. 

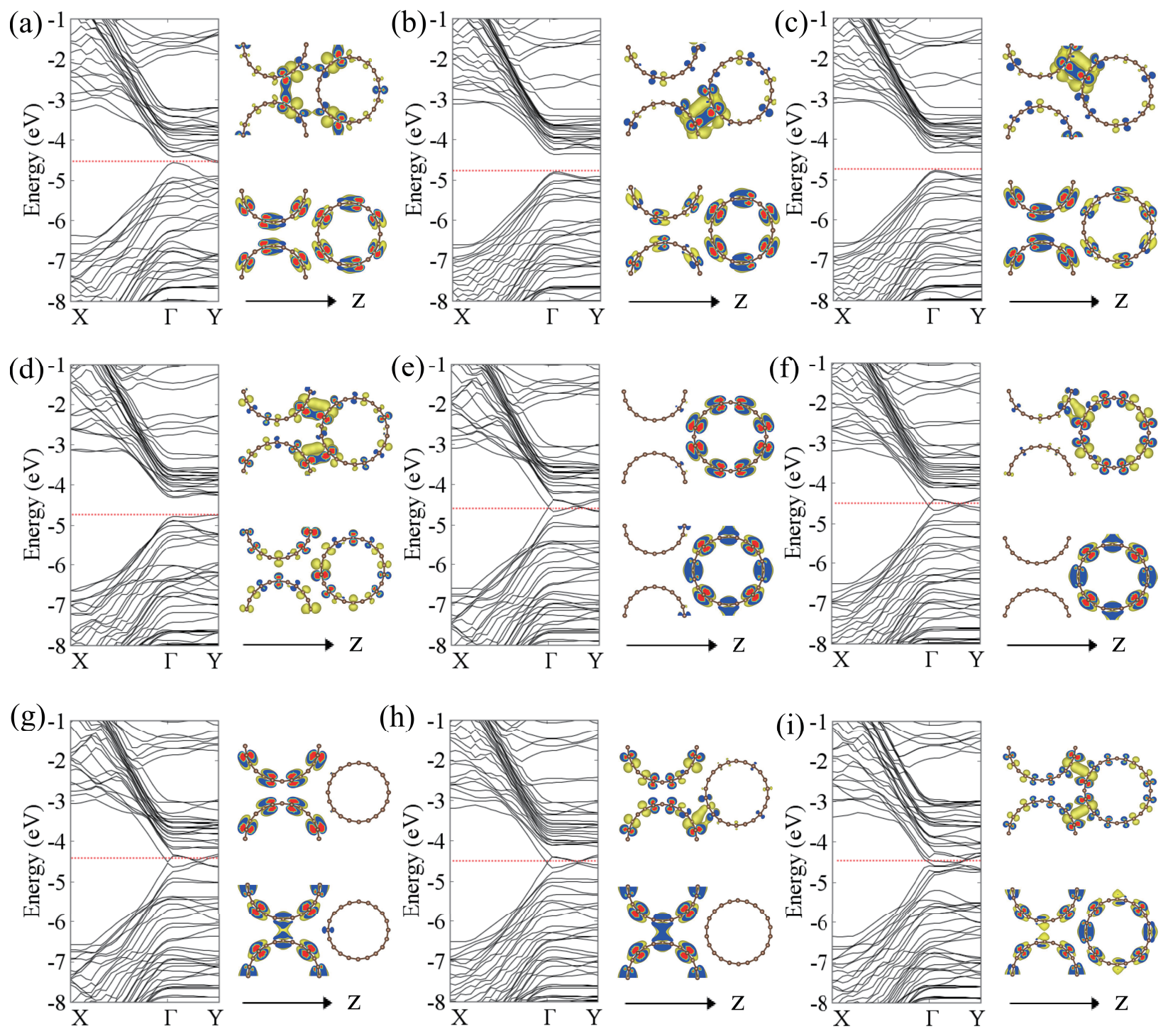

FIG. 5. Electronic energy band and squared wave function of the valence band top and the conduction band bottom of densely packed (a) $(10,0) /(10,0)$, (b) $(10,0) /(11,0)$, (c) $(11,0) /(10,0)$, (d) $(11,0) /(11,0)$, (e) $(12,0) /(10,0)$, (f) $(12,0) /(11,0),(\mathrm{g})(10,0) /(12,0),(\mathrm{h})(11,0) /(12,0)$, and (i) $(12,0) /(12,0)$ CNT thin films without an electric field. The energies are measured from that corresponding to the vacuum level situated at the cell boundary opposite to the electrode. Red dotted line approximately indicates the Fermi level. 
(a)

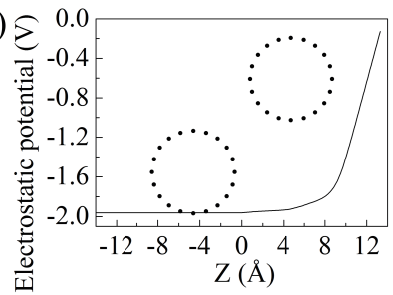

(c)

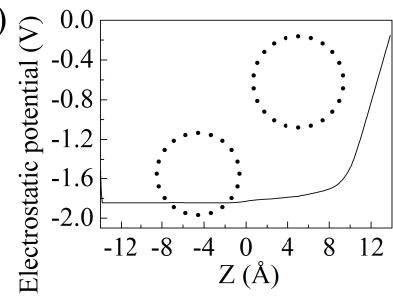

(e)

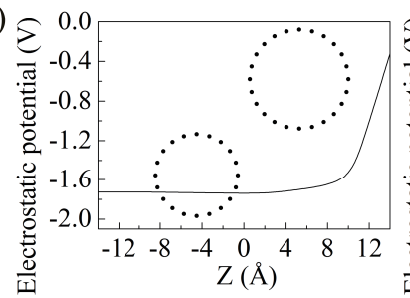

(g)

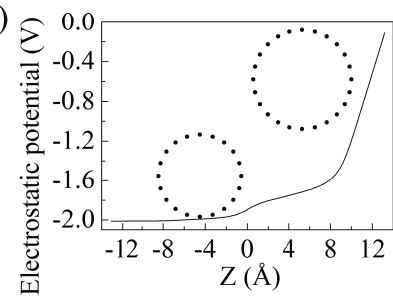

(i)

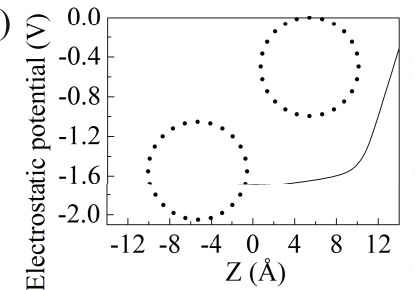

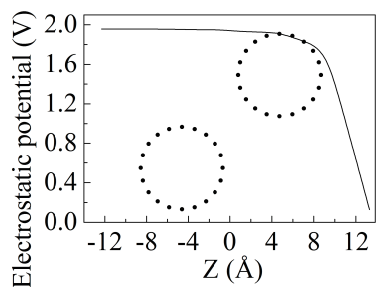
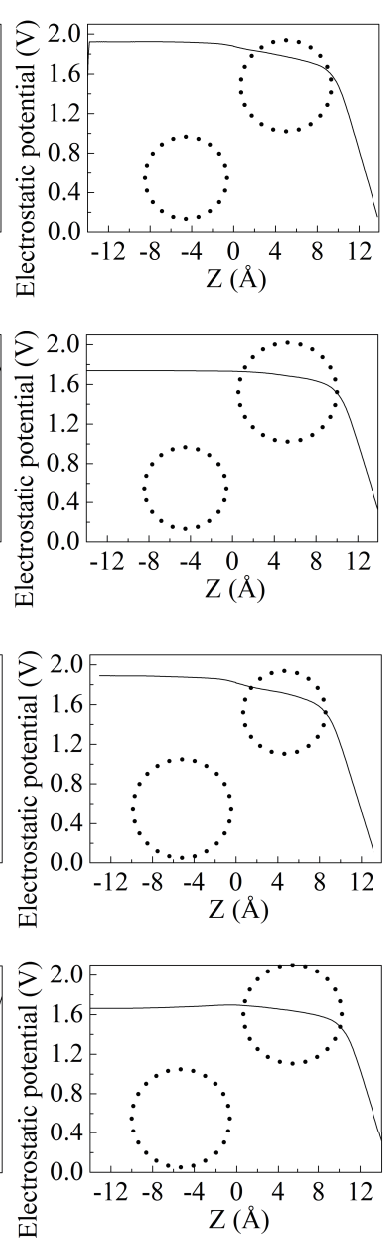

(b)
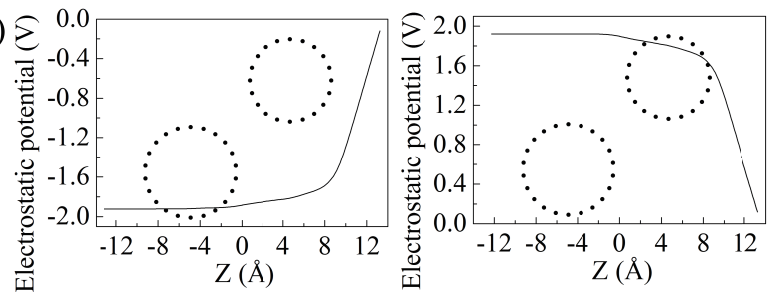

(d)
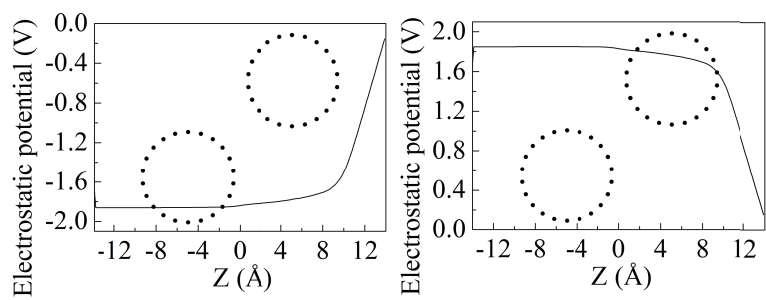

(f)
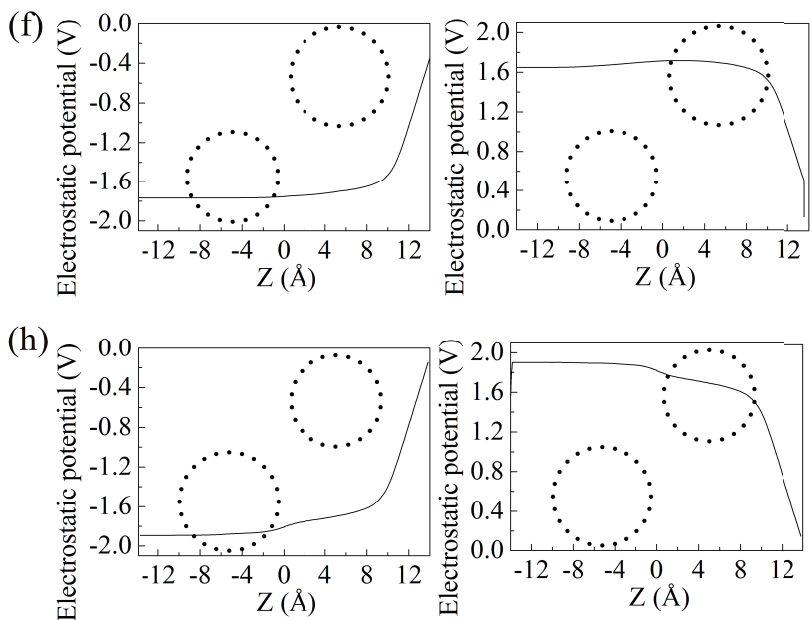

FIG. 6. Plane averaged electrostatic potential as a function of $z$ corresponding to the normal direction to the electrode in densely packed (a) $(10,0) /(10,0),(b)(10,0) /(11,0)$, (c) $(11,0) /(10,0)$, (d) $(11,0) /(11,0)$, (e) $(12,0) /(10,0)$, (f) $(12,0) /(11,0),(\mathrm{g})(10,0) /(12,0)$, (h) $(11,0) /(12,0)$, and (i) $(12,0) /(12,0)$ CNT thin films under an electric field. The electrode is situated at the right end of each figure. In each figure, left and right panels correspond to the carrier densities under the $0.1 \mathrm{~h}$ and $0.1 \mathrm{e}$ doping, respectively. 